Article

\title{
Applicability of Yeast Fermentation to Reduce Fructans and Other FODMAPs
}

\author{
Vera Fraberger ${ }^{\mathbb{D}}$, Lisa-Maria Call, Konrad J. Domig ${ }^{\mathbb{D}}$ and Stefano D'Amico *(D) \\ Department of Food Science and Technology, University of Natural Resources and Life Sciences (BOKU), \\ 1190 Vienna, Austria; vera.fraberger@boku.ac.at (V.F.); lisa.call@boku.ac.at (L.-M.C.); \\ konrad.domig@boku.ac.at (K.J.D.) \\ * Correspondence: stefano.damico@boku.ac.at; Tel.: +43-1-47654-75243
}

Received: 13 August 2018; Accepted: 4 September 2018; Published: 6 September 2018

\begin{abstract}
A diet low in fermentable oligosaccharides, disaccharides, monosaccharides and, polyols (FODMAPs) is recommended for people affected by irritable bowel syndrome (IBS) and non-coeliac wheat sensitivity (NCWS) in order to reduce symptoms. Therefore, the aim of this study was to evaluate the impact of 13 sourdough-related yeasts on FODMAP degradation, especially fructans. First, a model system containing a typical wheat carbohydrate profile was applied to evaluate the growth rate of each yeast strain. Additionally, changes in the sugar composition, for up to four days, were monitored by high-pressure anion-exchange chromatography (HPAEC). A more realistic approach with a wheat flour suspension was used to characterize $\mathrm{CO}_{2}$ production according to the Einhorn method. The reduction of the total fructans was analyzed using an enzymatic method. Furthermore, a fingerprint of the present fructans with different degrees of polymerization was analyzed by HPAEC. The results revealed strong differences in the examined yeast strains' ability to degrade fructans, in both the model system and wheat flour. Overall, Saccharomyces cerevisiae isolated from Austrian traditional sourdough showed the highest degree of degradation of the total fructan content and the highest gas building capacity, followed by Torulaspora delbrueckii. Hence, this study provides novel knowledge about the FODMAP conversion of yeast strains.
\end{abstract}

Keywords: FODMAPs; fructan; IBS; wheat; sourdough; yeasts; fermentation

\section{Introduction}

Current data indicates that irritable bowel syndrome (IBS) affects $7-15 \%$ of the population worldwide, with an estimated prevalence of $12 \%$ within Europe [1,2]. IBS is the most common chronic gastrointestinal disorder, which is defined as "a functional bowel disorder in which recurrent abdominal pain is associated with defecation or a change in bowel habits" [3]. Symptoms are abdominal pain, flatulence, diarrhea, constipation, intestinal cramps, nausea, and an altered gut microbiota [1-5], leading to a substantial reduction in the health-related quality of life (HRQOL) of patients [2]. Similar symptoms have been reported for people with non-coeliac wheat sensitivity (NCWS). Beside amylase-trypsin inhibitors (ATIs), wheat fructans are suspected to be the main triggers of symptoms [6,7]. Since no biomarkers are thus far available, and indications are similar to other disorders, reliable diagnoses are difficult [8].

However, it has been widely acknowledged that FODMAPs plays a major role in the pathological process of IBS and NCWS [9-11]. The term FODMAPs comprises fermentable oligosaccharides (fructans and galactans), disaccharides (lactose), monosaccharides (fructose), and polyols (sorbitol and mannitol), which are present in a number of grains, fruits, vegetables, and dairy products. A lack of enzymes results in the incomplete hydrolysis of glycosidic linkages in these complex polysaccharides, leading to malabsorption. In addition, the poorly absorbed short-chain carbohydrates 
are osmotically active, drawing more water into the intestine and affecting gut mobility. The undigested polysaccharides are transferred further to the large intestine, where the colonic microbiota rapidly ferments FODMAPs, causing diarrhea and gas production, symptoms consistent with IBS. Therefore, a diet low in FODMAPs has proven to be an effective approach to reduced symptoms in patients with both disorders $[8,9,12]$.

Wheat and its products are staple foods worldwide, accounting for a large extent of the daily consumption of FODMAPs. Moreover, they are the most important source of fructans in the Western European diet. Within FODMAPs, fructans belong to the class of fermentable oligosaccharides, contributing up to $70 \%$ to the daily fructan intake $[13,14]$. Several authors have already stated the influence of FODMAPs, and especially fructans, to trigger the symptoms of IBS and the importance of reducing its concentration in the diet of patients $[15,16]$.

In wheat flour, fructan concentrations range from 1.4-1.7 g/100 g, with whole-wheat flour containing 0.7-2.9 g/100 g [17]. Fifty-percent of fructans exhibit a degree of polymerization (DP) of 3,4 , and 5 (average of 4), with a maximum DP of 14-19, consisting of exclusively or mainly fructose units [14,18], and a maximum of one glucose unit per molecule can be present. Fructans deriving from cereals belong to the graminan type and are branched, which results in more complex structures compared to the inulin-type fructans found in vegetables [17,19].

Due to the high concentrations of fructans in wheat products, patients with IBS and NCWS should consume less FODMAP-containing products, as a single ingestion of approximately $0.3 \mathrm{~g} / \mathrm{kg}$ can trigger symptoms [20]. However, the authors of Ziegler et al. [21] reported a 77-90\% reduction of FODMAPs after yeast proofing for four hours, depending on the flour used. They reported a total reduction of raffinose, whereas fructans were degraded incompletely. Furthermore, this study revealed an excess of fructose. Additionally, Knez et al. [22] demonstrated a 40-60\% decrease in fructan content during the bread making process, where the fermentation time, present yeast strain, and yeast counts played a crucial role in the degradation process. The use of sourdough was proven to be one effective method to reduce the content of fructans in bread, as the invertase activity of yeasts degrades fructans [23]. An examination of the impact of traditional sourdough application on the reduction of fructan concentrations in bread showed a decrease of up to $0.06 \mathrm{~g} / 100 \mathrm{~g}$ [24].

Lactic acid bacteria and yeasts are present in traditional sourdough at a ratio of 10:1 to 1000:1, whereat these microorganisms lead to superior characteristics of sourdough [25-29]. The most prevalent yeast species in traditional sourdough are Saccharomyces cerevisiae, Candida humilis, Wickerhamomyces anomalus, Torulaspora delbrueckii, Kazachstania exigua, Pichia kudriavzevii, and Candida glabrata. Less frequent species include Kluyveromyces marxianus and Hanseniaspora uvarum [28,30]. Recent investigations [1,31] have already determined the probability of $K l$. marxianus strain CBS6014 to degrade fructans, showing a 90\% decrease of the initial fructan concentration due to dough fermentation for two hours. However, information about the applicability of further sourdough-relevant yeasts to degrade FODMAPs, especially fructans, and a comparison of different yeast strain processes is still lacking.

Therefore, the aim of this study was to investigate the potential of several yeast strains to reduce FODMAP levels, especially fructans. First, a model system with the typical carbohydrates found in wheat was applied to study the degradation of fructans and to monitor the metabolism of other carbohydrates. In addition, a more applied approach with wheat flour was used to obtain data on the gas production and reduction of graminan-type fructans.

\section{Materials and Methods}

\subsection{Applied Yeast Strains}

In total, 13 yeast strains were screened for their specific ability to ferment carbohydrates, especially for their potential to degrade fructans. Three strains were isolated from traditional Austrian sourdoughs and one from a baker's yeast; eight strains were obtained from either the 
BCCM or DSMZ databases. Table 1 lists all of the examined strains and their origin. Previously, the isolates obtained from traditional Austrian wheat or rye sourdoughs were identified by partial $26 \mathrm{~S}$ rDNA sequencing according to Waite et al. [32] and/or by MALDI-TOF MS (matrix-assisted laser desorption/ionization-time of flight mass spectrometry; MALDI Biotyper, Bruker Corporation). For identification by MALDI-TOF MS, the procedure was followed according to the manufacturer's manual for the extraction method. Table S1 reports the sequencing and MALDI-TOF MS results.

Table 1. Yeast species, strain information, and source of isolation used for the determination of the potential to degrade FODMAPs.

\begin{tabular}{ccc}
\hline Species & Strain & Source of Isolation \\
\hline Candida glabrata & BCCM/MUCL ${ }^{1} 51245$ & wheat sourdough, mill D12 \\
Candida humilis & BCCM/MUCL ${ }^{1} 30041$ & San Francisco sourdough \\
Kazachstania exigua & BCCM/MUCL ${ }^{1} 52365$ & brewing, Japan \\
Kluyveromyces marxianus & BCCM/MUCL ${ }^{1} 30016$ & - \\
Pichia kudriavzevii & BCCM/MUCL ${ }^{1} 29043$ & industrial sourdough, France \\
Torulaspora delbrueckii & BCCM/MUCL ${ }^{1} 51211$ & wheat and rye sourdough, artisan bakery, Belgium \\
Torulaspora pretoriensis & BCCM/MUCL ${ }^{1} 27827$ & soil, South Africa \\
Wickerhamomyces anomalus & DSM ${ }^{2} 6766$ & - \\
Candida lambica & isolate & rye sourdough, Austria \\
Hanseniaspora uvarum & isolate & wheat sourdough, Austria \\
Saccharomyces cerevisiae & isolate & baker's yeast \\
Saccharomyces cerevisiae & isolate & rye sourdough, Austria \\
Torulaspora delbrueckii & isolate & wheat sourdough, Austria \\
\hline 1 BCCM/MUCL: Belgian Coordinated Collection of Microorganisms / Agro-food and Environmental Fungal \\
Collection. ${ }^{2}$ DSMZ: Leibniz-Institute DSMZ-German Collection of Microorganisms and Cell Cultures.
\end{tabular}

\subsection{Preparation and Growth of Yeast Cells in a Model System}

For the model system, a typical carbohydrate profile found in wheat grains and flours was used. The concentrations of sugars and fructans were set according to the results obtained by Call et al. [33] and are presented in Table 2. To obtain a more realistic profile, sucrose and maltose were added, which are usually included intentionally or are produced by amylases in wheat dough. The appropriate amount of tryptone was dissolved in $\mathrm{H}_{2} \mathrm{O}_{\text {dest }}$ and autoclaved at $121{ }^{\circ} \mathrm{C}$ for 15 min at one1 bar overpressure. The dissolved carbohydrate solution was filter sterilized through a $0.2-\mu \mathrm{m}$ polyamide membrane (VWR International GmbH, Darmstadt, Germany) and added to the tryptone solution.

Table 2. Medium composition of the model system [33].

\begin{tabular}{|c|c|c|c|}
\hline & Ingredient & Supplier & {$[\mathrm{g} / 100 \mathrm{~mL}]$} \\
\hline \multirow{6}{*}{$\begin{array}{l}\text { Natural ratios occurring } \\
\text { in flour }\end{array}$} & D-Glucose & \multirow{5}{*}{$\begin{array}{c}\text { Carl Roth GmbH + Co. KG, } \\
\text { Karlsruhe, Germany }\end{array}$} & 0.02 \\
\hline & D-Fructose & & 0.04 \\
\hline & Sucrose & & 0.41 \\
\hline & Raffinose pentahydrate & & 0.17 \\
\hline & Maltose monohydrate & & 0.04 \\
\hline & $\begin{array}{l}\text { Fructooligosaccharides } \\
\text { from chicory (max. DP 8) }\end{array}$ & Megazyme, Bray, Ireland & 1.37 \\
\hline \multirow{3}{*}{ Supplements } & Maltose monohydrate & \multirow{2}{*}{$\begin{array}{c}\text { Carl Roth GmbH + Co. KG, } \\
\text { Karlsruhe, Germany }\end{array}$} & 0.64 \\
\hline & Sucrose & & 0.4 \\
\hline & Tryptone & Oxoid LTD, Hampshire, England & 0.6 \\
\hline
\end{tabular}

The yeast species were routinely cultivated in worth broth (Merck KGaA, Darmstadt, Germany) and incubated for $48 \mathrm{~h}$ at $25^{\circ} \mathrm{C}$. For starvation of the cells, the biomass was transferred to tryptone $(6 \mathrm{~g} / \mathrm{L}$; Oxoid Ltd., Hampshire, UK) at a dilution ratio of 1:100. After $24 \mathrm{~h}$, the medium, together with 
starved yeast cultures (1:100) was added in duplicate to Honeycomb format plates (Bartelt GmbH, Graz, Austria). Turbidity data for growth curves of yeast species were analyzed using the automated density monitoring system BioscreenC analyze reader (Oy Growth Curves Ab Ltd., Helsinki, Finland). Reading for $96 \mathrm{~h}$ at $25{ }^{\circ} \mathrm{C}$, measurements were taken at an optical density of $600 \mathrm{~nm}\left(\mathrm{OD}_{600}\right)$ every 10 min after shaking.

\subsection{Gas Production Measurement According to Einhorn}

The $\mathrm{CO}_{2}$ building capacity of yeasts were measured according to the method of Einhorn. Whole-wheat flour from the Austrian variety Arnold was used. Kernels of this variety were collected from nine different locations in Austria (research fields of AGES) over two years (2016 and 2017) to produce a standardized flour. A total of $1 \mathrm{~g}$ of flour was mixed with $10 \mathrm{~mL}$ of sterilized water and inoculated with the listed yeast strains (Table 1). Yeast counts were in the range of $\log 7 \mathrm{CFU} / \mathrm{mL}$ (detailed yeast counts are presented in Table S2). The yeast-containing wheat flour suspensions were transferred to fermentation locks according to Einhorn. The $\mathrm{CO}_{2}$ building capacity was read in $\mathrm{mL}$ every $15 \mathrm{~min}$ for up to $8 \mathrm{~h}$ and $15 \mathrm{~min}$. Figure $\mathrm{S} 1$ gives examples of the fermentation locks.

\subsection{FODMAP Extraction of the Model System}

To determine FODMAP conversion in the carbohydrate model system, 4 measuring intervals were defined based on the results of the previous growth experiments- $6 h, 24 h, 48 h$, and $72 \mathrm{~h}$ of incubation. The respective cell mass was transferred from the Honeycomb format plates into $2 \mathrm{~mL}$ centrifugation tubes. Proteins were removed by Carrez-precipitation. Hence, $15 \mu \mathrm{L}$ of Carrez I solution-potassium hexacyanoferrate(II)trihydrate (Merck, Darmstadt, Germany) in $1000 \mathrm{~mL}$ $\mathrm{H}_{2} \mathrm{O}$, and $15 \mu \mathrm{L}$ of Carrez II Solution-300 g zinc acetate dehydrate (VWR International GmbH, Darmstadt, Germany) in $1000 \mathrm{~mL} \mathrm{H}_{2} \mathrm{O}$ was added to precipitate the proteins. After centrifugation at $16,000 \times g$ for $30 \mathrm{~min}$ at $4{ }^{\circ} \mathrm{C}$, the supernatant was transferred to a volumetric flask and filled up to $1 \mathrm{~mL}$. Next, the solution was filtered through a $0.2-\mu \mathrm{m}$ filter (Rotilabo Mini-Tip syringe filter; Carl Roth $\mathrm{GmbH}+\mathrm{Co}$. KG, Karlsruhe, Germany) and diluted to receive carbohydrate concentrations between 5-200 ppm and transferred into vials $(1.5 \mathrm{~mL}$ clear glass; VWR International $\mathrm{GmbH}$, Darmstadt, Germany) for further analysis.

\subsection{Extraction of Graminan-Type Fructans}

To determine changes of DP of graminan-type fructans after fermentation, a fingerprint analysis was performed after extraction of the wheat flour-water suspension. Following fermentation for $8 \mathrm{~h}$ and $15 \mathrm{~min}$, the suspensions in the Einhorn locks were subjected to an ethanol extraction. The matrix was transferred into a centrifugation tube, $30 \mathrm{~mL}$ of $96 \%$ ethanol (VWR International GmbH, Darmstadt, Germany) was added and the mixture was heated to $80^{\circ} \mathrm{C}$ for $20 \mathrm{~min}$. After centrifugation at $9000 \times g$ for $6 \mathrm{~min}$, the supernatant was filled up to the defined volume of $50 \mathrm{~mL}$. A $3 \mathrm{~mL}$ aliquot of the extract was evaporated at $50{ }^{\circ} \mathrm{C}$ under a nitrogen stream to dryness and resuspended in $0.96 \mathrm{~mL}$ of water. Twenty $\mu \mathrm{L}$ each of amyloglucosidase (AMG) and alpha-amylase (Novozymes, Ireland) were added, and incubation was carried out at $50{ }^{\circ} \mathrm{C}$ for $30 \mathrm{~min}$. Afterwards, protein precipitation, as described in Section 2.4, was conducted. The solution was sterile-filtered through a $0.2-\mu \mathrm{m}$ filter (Rotilabo Mini-Tip syringe filter; Carl Roth GmbH + Co. KG, Karlsruhe, Germany) and used further for HPAEC-PAD (high pressure anion-exchange chromatography-pulsed amperometric detection) analysis.

\subsection{FODMAP and Fructan Analysis}

To determine the FODMAP concentrations and the DP of fructans, HPAEC with a Carbopac PA210 column $(2 \times 150 \mathrm{~mm})$ from ThermoFisher Scientific (Sunnyvale, CA, USA) was applied at a flow rate of $0.15 \mathrm{~mL} / \mathrm{min}$. A gradient elution with $150 \mathrm{mM} \mathrm{NaOH}$ and $150 \mathrm{mM} \mathrm{NaOH} / 500 \mathrm{mM}$ Na-acetate was used on a DionexTM ICS-5000+ System (ThermoFisher Scientific, Sunnyvale, CA, USA) for separation. The concentrations of glucose, fructose, sucrose, maltose, raffinose, and fructans were determined by 
pulsed amphoteric detection (PAD). A gold electrode with a carbohydrate waveform was used for electrochemical detection according to the manufacturer's instructions. For integration and calibration, the software Chromeleon 7 was utilized. The method was calibrated in a range from $0.1-25 \mathrm{mg} / \mathrm{L}$ with glucose, fructose, sucrose, maltose, raffinose ( $\geq 99.5 \%$ purity; Carl Roth $\mathrm{GmbH}+\mathrm{Co}$. KG, Karlsruhe, Germany), and small fructans from DP 3-5 (Megazyme, Bray, UK). For comparison, a fructan standard with up to DP 8 was also analyzed (fructooligosaccharide P-FOS28, Megazyme, Bray, UK). The qualitative reduction of the total graminan-type fructans, present in the wheat flour suspensions, was determined by an enzymatic-spectrophotometric method according to AOAC standard 999.3.

\subsection{Statistical Analysis}

The data interpretation was performed with SPSS software (IBM). The correlation test according to Pearson was applied to compare the fructan conversion results gained by the enzymatic assay and HPAEC-PAD.

\section{Results}

\subsection{Yeast Growth}

The cell growth of 13 different yeast strains were evaluated for up to $96 \mathrm{~h}$ at $25{ }^{\circ} \mathrm{C}$ with an automated density monitoring system using a model medium, containing a typical carbohydrate profile found in whole-grain wheat. The growth curves are plotted in Figure 1.

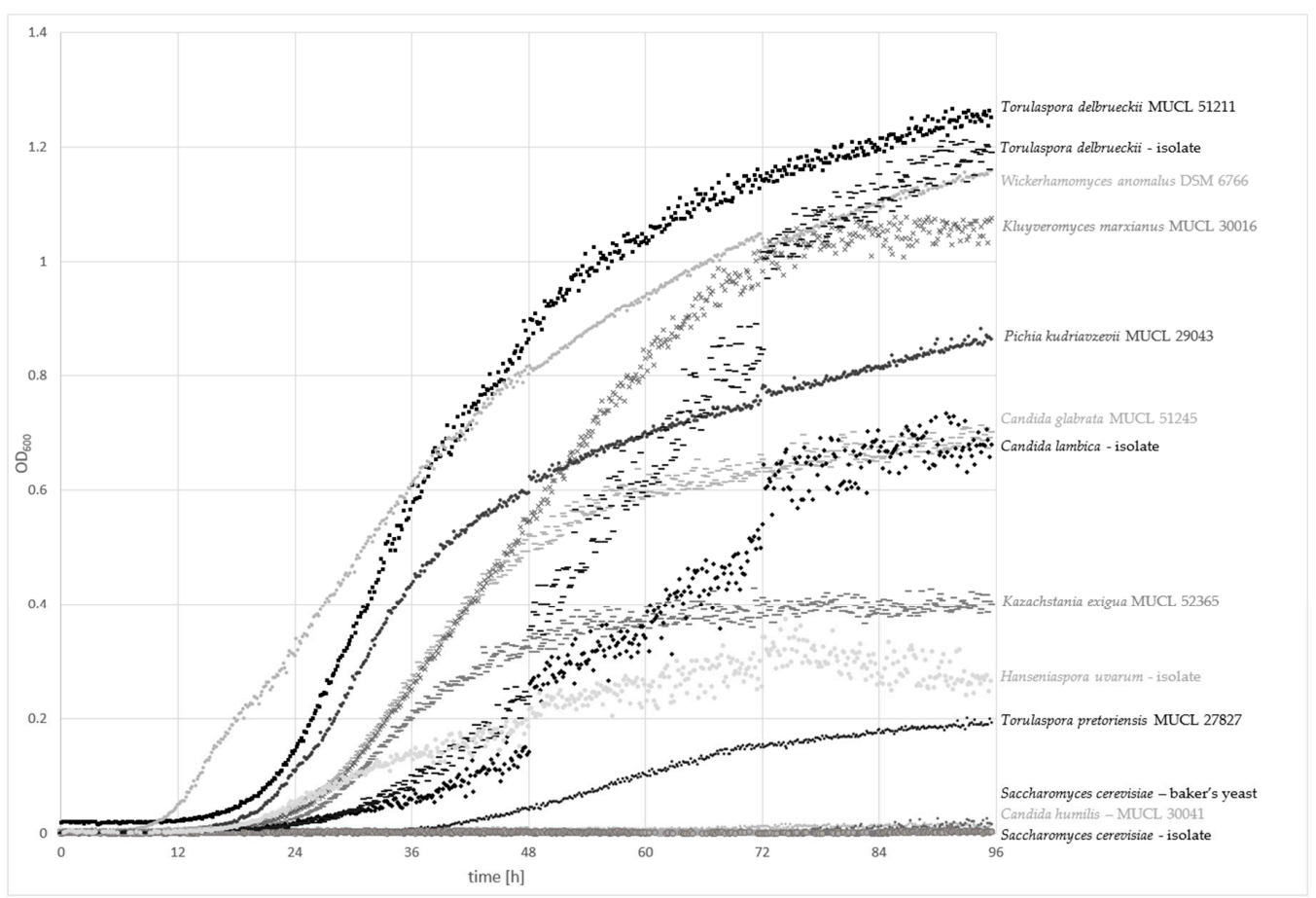

Figure 1. Measurement of the optical density at $600 \mathrm{~nm}\left(\mathrm{OD}_{600}\right)$ over $96 \mathrm{~h}$ of incubation at $25{ }^{\circ} \mathrm{C}$ to indicate the growth rate of sourdough-relevant yeasts in a model medium containing mono-/di-/trisaccharides, and fructans, according to the average concentrations found in 20 Austrian wheat varieties (Table 1).

Torulaspora delbrueckii strains (MUCL 51211 and the sourdough isolate) exhibited the highest optical density at $600 \mathrm{~nm}\left(\mathrm{OD}_{600}\right)$ of 1.3-1.2 nm, respectively, after $96 \mathrm{~h}$ of incubation, followed by Wickerhamomyces anomalus DSM 6766 and Kluyveromyces marxianus MUCL 30016. Pichia kudriavzevii MUCL 29043, Candida glabrata MUCL 51245, and the sourdough isolate C. lambica showed $\mathrm{OD}_{600}$ values ranging from 0.9-0.7 nm. Kazachstania exigua MUCL 52365, the isolate Hanseniaspora uvarum, 
and Torulaspora pretoriensis MUCL 27827 exhibited values between 0.4 and $0.2 \mathrm{~nm}$. Only Saccharomyces cerevisiae isolates (baker's yeast and the sourdough isolate) and C. humilis MUCL 30041 showed no significant increase in the $\mathrm{OD}_{600}$.

A lag phase shorter than $12 \mathrm{~h}$ was only achieved by W. anomalus DSM 6766. Exponential growth after 18-20 h was observed for T. delbrueckii and P. kudriavzevii MUCL 29043. The other strains started the log phase after a period of more than 20 h. T. pretoriensis MUCL 27827 showed slow growth, and a moderate increase of $\mathrm{OD}_{600}$ was detected after over $36 \mathrm{~h}$. Generally, the strains with the fastest growth rates had the highest $\mathrm{OD}_{600}$ values. Nevertheless, some exceptions were observed. T. delbrueckii isolated from sourdough achieved high final $\mathrm{OD}_{600}$ values, although the log phase started after approximately 24 h. K. exigua MUCL 52356 accomplished the lag phase after about 20 h, whereas only medium-range $\mathrm{OD}_{600}$ values were achieved after 96 h. H. uvarum exhibited similar growth behavior, but the final $\mathrm{OD}_{600}$ was low in comparison to K. exigua MUCL 52356.

\section{2. $\mathrm{CO}_{2}$ Formation Properties}

The $\mathrm{CO}_{2}$ production capacity of 13 yeast strains were tested using fermentation locks according to Einhorn (Figure S1). The data on the $\mathrm{CO}_{2}$ production rates $\left(\mathrm{mL} \mathrm{CO}_{2} / \mathrm{min}\right)$ of $\mathrm{CO}_{2}$-positive yeast strains are presented in Figure 2. S. cerevisiae strains exhibited the highest $\mathrm{CO}_{2}$ formation capacity $(1.8 \mathrm{~mL})$ after $8 \mathrm{~h}$ and $15 \mathrm{~min}$ of incubation at $25^{\circ} \mathrm{C}$, followed by T. delbrueckii MUCL 51211 with a $\mathrm{CO}_{2}$ production of $0.8 \mathrm{~mL}$. K. exigua MUCL 52365 and C. glabrata MUCL 51245 exhibited poor formation capacities of $0.2 \mathrm{~mL}$. Further tested strains C. humilis MUCL 30041, Kl. marxianus MUCL 30016, P. kudriavzevii MUCL 29043, T. pretoriensis MUCL 27827, W. anomalus DSM 6766, and sourdough isolates P. fermentans, C. lambica, T. delbrueckii, and H. uvarum had no observable $\mathrm{CO}_{2}$ production.

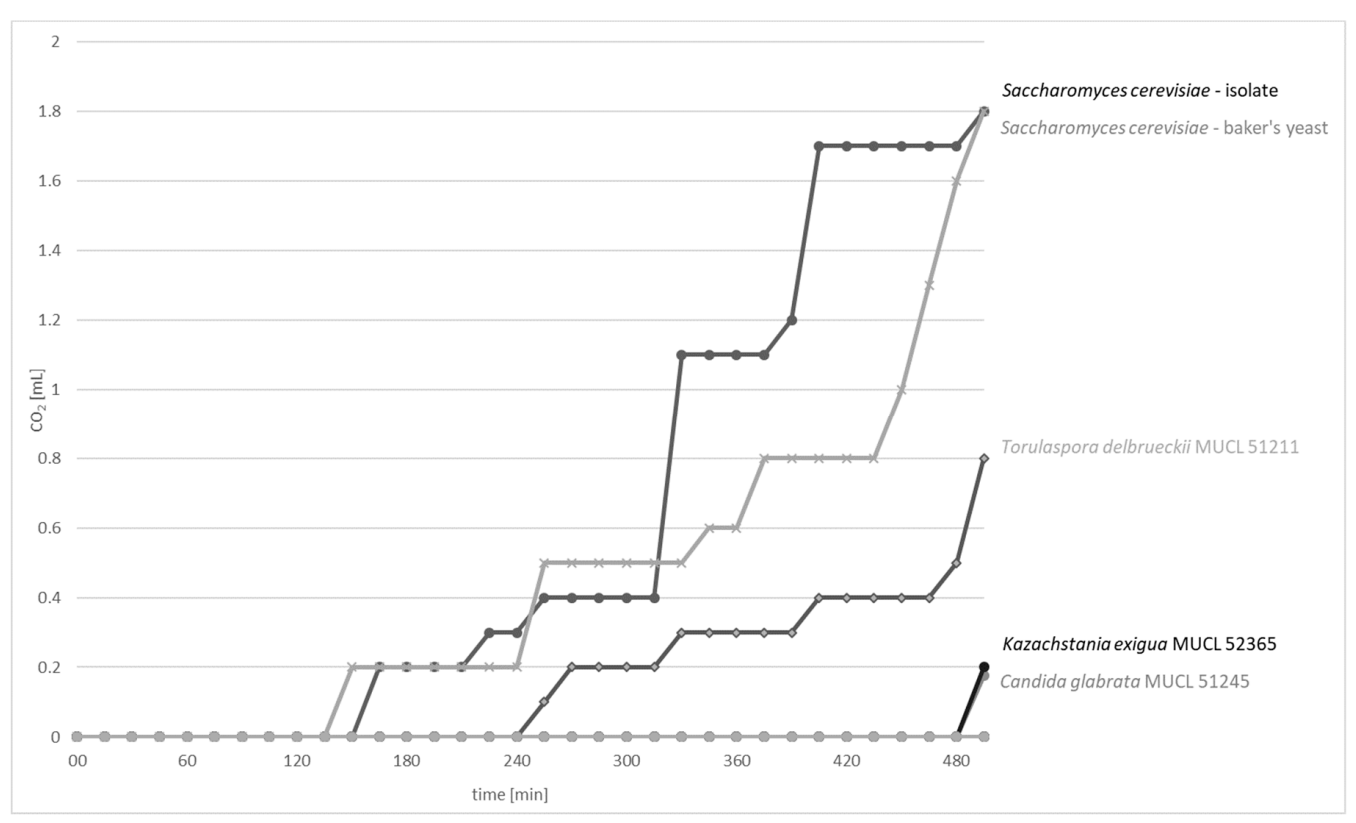

Figure 2. Time course of $\mathrm{CO}_{2}$ production $(\mathrm{mL} \mathrm{CO} / \mathrm{min})$ of $\mathrm{CO}_{2}$-positive yeast strains $S$. cerevisiae, T. delbrueckii MUCL 51211, K. exigua MUCL 52365, and C. glabrata MUCL 51245 over $8 \mathrm{~h}$ and 15 min of incubation at $25^{\circ} \mathrm{C}$.

For $\mathrm{CO}_{2}$-positive yeast strains, the graphs showed mainly constant levels during the period of 30-90 min, followed by an observable strong and sudden increase. This effect can be explained by the production of small $\mathrm{CO}_{2}$ bubbles, which were first entrapped within the wheat flour suspension. After they have aggregated to bigger bubbles, the volume of $\mathrm{CO}_{2}$ was measureable as the bubbles were able to displace the suspension and rise to the top. However, although the curves of the S. cerevisiae strains looked different, similar gas productions can be assumed because of these circumstances. 


\subsection{Conversion of Carbohydrates by Yeasts in the Model System}

The fermentation profile of 13 different yeast strains were examined over three days. After four different time periods ( 6 h, 24 h, 48 h, and 72 h), samples were taken and analyzed to evaluate changes in the carbohydrate profile, i.e., the degradation or production of glucose, sucrose, fructose, raffinose, maltose, and fructans (DP 4-8). The results are illustrated in Figure 3 as pie charts, showing the relative abundance of quantified sugars and fructans based on HPAEC-PAD analysis.

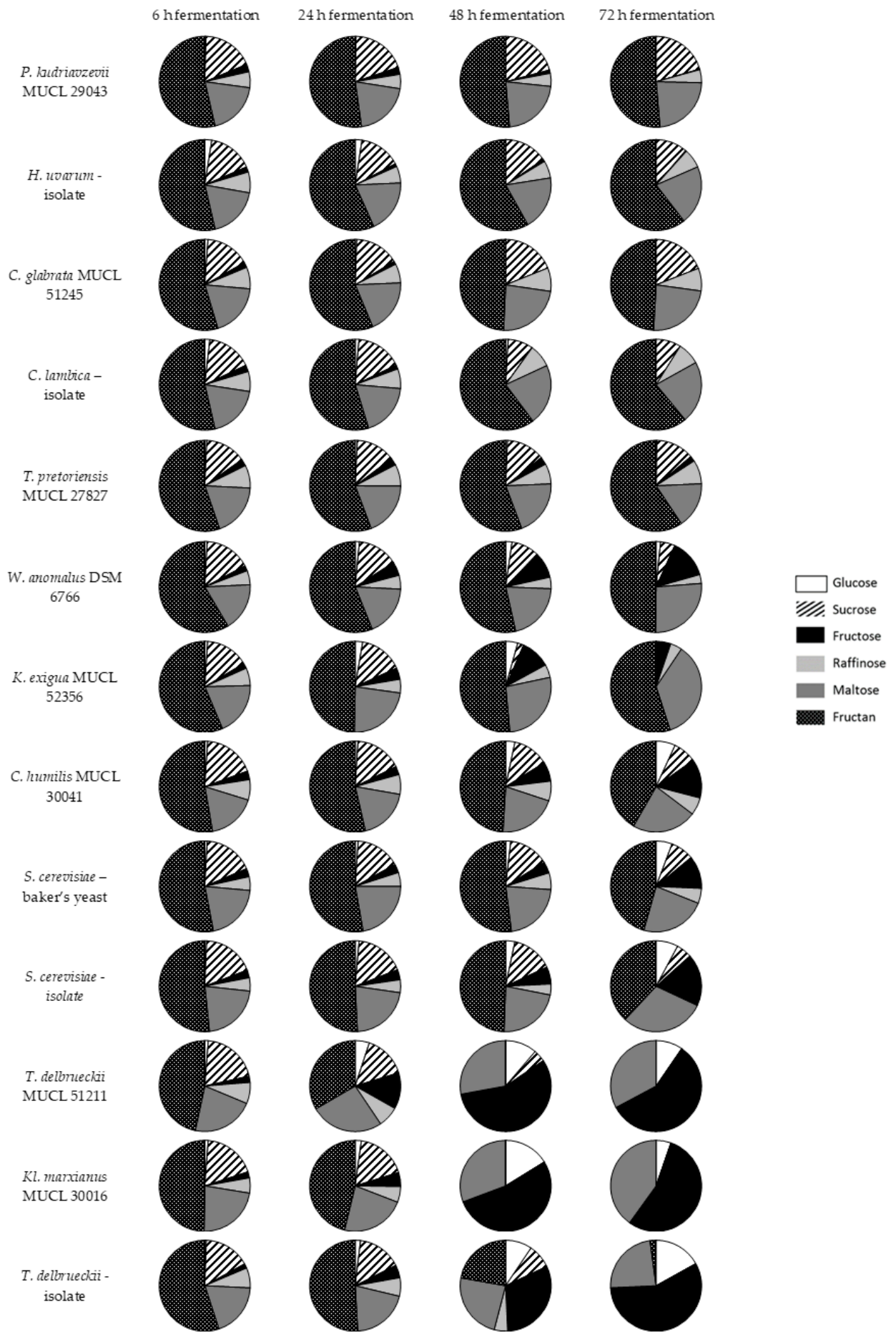

Figure 3. Fermentation profile of 13 yeasts strains over 4 measuring points, $6 \mathrm{~h}, 24 \mathrm{~h}, 48 \mathrm{~h}$, and $72 \mathrm{~h}$ of incubation at $25^{\circ} \mathrm{C}$ in a model system; examined carbohydrates: Glucose, sucrose, fructose, raffinose, maltose, and fructan. 
Of the 13 tested yeasts, P. kudriavzevii MUCL 29043, C. glabrata MUCL 51245, T. pretoriensis MUCL 27827, and the sourdough isolates H. uvarum and C. lambica were not able to reduce fructan levels to a relevant degree. Furthermore, the concentration of raffinose remained constant, which indicted that these strains were not able to consume this trisaccharide. The small amount of initial glucose were digested quite fast, and fructose slightly slower. Among these strains, only T. pretoriensis MUCL 27827 showed fructose residues after three days. The maltose concentrations remained almost unchanged. Sucrose was fermented to a small degree by T. pretoriensis MUCL 27827, H. uvarum and C. lambica, whereas sucrose levels were not significantly affected by the other yeast strains of this group.

A second group consisted of strains with moderate fructan conversion including K. exigua MUCL 52356, C. humilis MUCL 30041, W. anomalus DSM 6766, and both S. cerevisiae strains. These yeasts were able to degrade fructans. However, after three days of fermentation, high fructan levels still remained. Raffinose was degraded only partially by the examined yeast strains, with one exception - the isolate of S. cerevisae completely converted this trisaccharide. Due to depolymerisation of the oligosaccharides, the content of monosaccharides had risen. In particular, the concentration of fructose increased markedly, and glucose increased to a lesser extent. Maltose was not fermented in notable amounts and remained more or less constant. Due to the reduction of other sugars, the relative abundance of maltose could actually increase, as was illustrated for K. exigua MUCL 52356. Sucrose was fermented incompletely, as low sucrose concentrations were detected after three days.

Both of the T. delbrueckii strains, and Kl. marxianus MUCL 30016 demonstrated the best fructan degradation. In addition, the highest amounts of fructose were produced by these strains. Kl. marxianus MUCL 30016 and T. delbrueckii MUCL 51211 were able to completely ferment fructans after two days; a reduction in the fructose and glucose content from $48-72 \mathrm{~h}$ of incubation was observable for these yeasts as well. The T. delbrueckii isolate degraded fructans more slowly, and small fructans residues were detected. Raffinose and sucrose were digested entirely. Maltose was fermented only to a minor extent and persisted more or less unchanged. Again, due to the reduction of other sugars, a relative increase in maltose was seen, which can be explained by presenting the relative abundance as pie charts.

\subsection{Fructan Degradation by Yeasts in Wheat Flour}

Chromatograms of fructan fingerprints, showing the distribution of DP, from two selected strains with the best and worst ability for fructan degradation are presented in Figure 4. The overall results of the 13 yeast strains analyzed by HPAEC-PAD are available within the supplementary material (Figure S3).
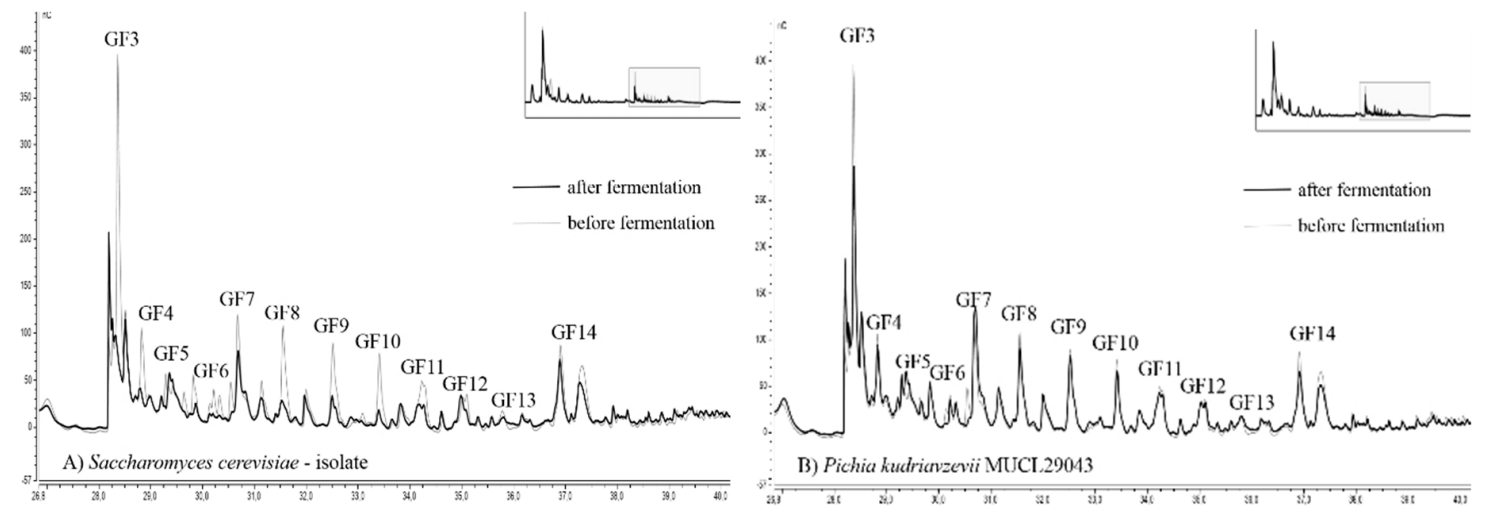

Figure 4. HPAEC (high pressure anion-exchange chromatography) fingerprint of fructans fermented by (A) Saccharomyces cerevisiae-sourdough isolate, and (B) Pichia kudriavzevii MUCL 29043. Chromatograms showing the initial fructan profile (grey line) and the alteration after $8 \mathrm{~h}$ and $15 \mathrm{~min}$ of fermentation at $25^{\circ} \mathrm{C}$ (black line) and are illustrated for both strains. G (glucose) and F (fructose).

To evaluate the alteration of the fructan profile ranging from DP 4-16, the fingerprint before and after fermentation is illustrated. The DP of fructans was estimated based on their retention times 
according to Verspreet et al. [34]. Due to the removal of starch-based dextrins by amylases, all detected peaks can be assigned to fructans.

C. humilis MUCL 30041, C. glabrata MUCL 51245, and P. kudriavzevii MUCL 29043 exhibited the lowest degree of alteration compared to the initial fructan fingerprint. The adulteration of fructans, as shown by HPAE chromatography, was very low. Only the smallest fructans with DP 4 were reduced in noticeable amounts. The height of all other peaks was reduced only to a small degree. T. delbrueckii MUCL 51211, S. cerevisiae-baker's yeast, Kl. marxianus MUCL 30016, W. anomalus DSM 6766, T. pretoriensis MUCL 27827, and the sourdough isolates C. lambica and H. uvarum exhibited moderate degradation of fructans. The most significant changes were observed for K. exigua MUCL 52365 and the sourdough isolates S. cerevisiae and T. delbrueckii (Figure 4). Fingerprints of these strains revealed severe reductions of peaks until DP 11 (GF 10). The fructans with higher molecular weights showed less adulteration in peak height. These findings were confirmed by other studies, which reported that smaller fructans were consumed more efficiently than those with higher DP [35].

In addition to the qualitative characterization of fructans by HPAEC, the samples from the $\mathrm{CO}_{2}$ production tests were analyzed by an enzymatic method to evaluate the decrease of fructan concentration. The reduction of total fructan content after $8 \mathrm{~h}$ and $15 \mathrm{~min}$ of incubation at $25^{\circ} \mathrm{C}$ was measured according to AOAC standard 999.3. To calculate the decrease of fructose oligomers, the initial content and the amount of fructans after fermentation were quantified. The reductions in fructan content (\%) due to fermentation of single yeast strains are presented in Figure 5. With a decrease of the total fructan content of 10-30\%, Candida spp., H. uvarum, T. pretoriensis MUCL 27827, and W. anomalus DSM 6766 showed the lowest capacity for FODMAP reduction of the tested yeast species. K. exigua MUCL 52365 revealed a total fructan reduction of approximately 50\%. S. cerevisiae-baker's yeast, the T. delbrueckii stains, and Kl. marxianus MULC 30016 revealed a decrease in the total fructan content between $60-80 \%$. With a reduction of $83 \%$, the sourdough isolate of S. cerevisiae had the highest decrease in fructans.

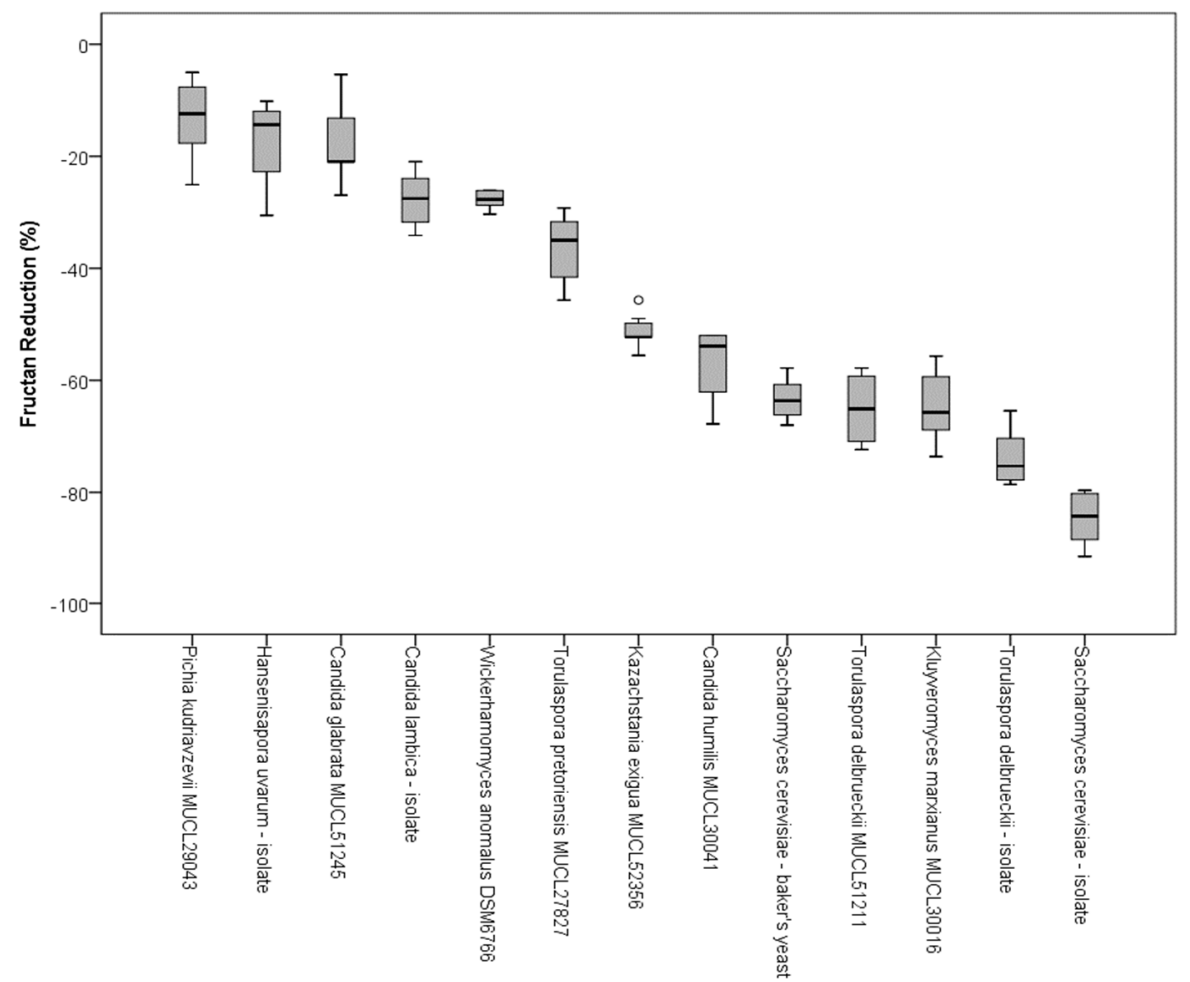

Figure 5. Box plots of the fructan reduction of examined yeast strains in wheat flour after $8 \mathrm{~h}$ and $15 \mathrm{~min}$ of incubation at $25^{\circ} \mathrm{C}$, determined according to the AOAC 999.3 standard; circles present outliners. 


\section{Discussion}

This study provides novel findings regarding the FODMAP reduction during fermentation by yeasts. First, the degradation potential of 13 yeast strains related to traditional sourdough were evaluated using a model system containing small fructooligosaccharides (max. DP 8) from chicory. Second, the alteration of graminan-type fructans in wheat flour was investigated using a yeast-mediated fermentation procedure. For examination of the leavening capacity, the $\mathrm{CO}_{2}$ building potential was determined according to Einhorn.

\subsection{Conversion Dynamics of Carbohydrates by Yeasts in the Model System and in Wheat Flour}

An assessment of the degradation and consumption of saccharides in a model system revealed very different capacities of the examined yeast strains. The chosen sugar profile, together with short-chain fructans (until DP 8), was suitable for the evaluation of fructan degradation and consumption of sugars found in wheat flour. Due to the simple fructan profile, a fast and accurate quantification by HPAEC was possible. The addition of maltose and sucrose provided a realistic system that closely matched atypical wheat dough. The established model system classified the examined yeast strains into three groups: Yeasts with poor, moderate, and superior fructan degradation. Similar results were obtained for the fermentation experiments of $\mathrm{CO}_{2}$ production within a wheat flour suspension. Again, three different groups regarding the degree of fructan hydrolysis could be defined.

Correlation analysis according to Pearson revealed a high consistency between the two systems. A highly significant ( $p$-level of 0.01) relationship with an $R^{2}$ of 0.59 was observed between the enzymatically measured fructan hydrolysis in wheat flour and the remaining fructan levels in the model system after fermentation for $72 \mathrm{~h}$. Obviously, a stronger correlation was prevented due to the different time periods of fermentation (the model system was $72 \mathrm{~h}$ compared to approximately $8 \mathrm{~h}$ for the fermentation experiments with wheat flour). A second reason for the low correlation could be the different growth rates of the examined yeast strains, especially with respect to both $S$. cerevisiae strains. These strains showed very low $\mathrm{OD}_{600}$ values and thus poor growth. Furthermore, fructans were hydrolysed to a lower extent, compared to the setup with wheat flour. Excluding the data from both of the $S$. cerevisiae strains revealed an increased correlation, with an $R^{2}$ of 0.83 between the fermented wheat flour suspension and the model system.

Moreover, the conversion and consumption of other sugars could be evaluated by the model system. Each strain fermented sucrose, whereat for C. glabrata MUCL 51245, the lowest sucrose decrease of $26 \%$ was observed. One exception was P. kudriavzevii MUCL 29043, where an increased concentration of $6 \%$ was measured due to partial fructan hydrolysis. These results were in accordance with previous studies [36]. Raffinose was metabolized by the majority of the examined yeasts. Some strains showed no or only minor breakdown of raffinose, which could arise from small variations due to the determination method used. A comparison with the literature verified this assumption for Candida spp. and P. kudriavzevii [36]. Controversial findings were found in respect to K. exigua [36]. Furthermore, relationships between fructan degradation and the consumption of raffinose and sucrose were found. Yeast strains, which depolymerized fructans to a large extent, consumed raffinose and sucrose as well, and invertase and inulinase are responsible for releasing the fructose moiety of these carbohydrates [37].

According to the literature [36], each tested yeast strain is able to ferment glucose, which was confirmed by this study as well. However, the consumption was partly masked due to the production of glucose, as it was released during the decomposition of fructans, sucrose, and raffinose. This effect was also observable for fructose concentrations, which increased as the fructans and raffinose were degraded. However, for species exhibiting the highest potential to depolymerize fructans (T. delbrueckii MUCL 51211 and Kl. marxianus MUCL 30016), a decrease in fructose was apparent after $72 \mathrm{~h}$ of incubation. This is consistent with studies showing the capacity of T. delbrueckii and Kl. marxianus to degrade fructose $[38,39]$. 
As previously mentioned, invertase and inulinase are able to depolymerize fructans. However, large polysaccharides barely pass the cell wall and thus must be hydrolysed outside of the cell [40]. The invertase produced by $S$. cerevisiae is retained inside the cell wall, whereas inulinase, produced by Kl. marxianus, is partly secreted [40]. This might explain why Kl. marxianus degrades fructans faster and to a higher degree than S. cerevisiae. The chosen system and sugar concentrations were not suitable to evaluate maltose conversion, because other sugars were present in excess during the evaluated time period. Nevertheless, maltose-positive strains [36], such as baker's yeast, T. delbrueckii, and $W$. anomalus, showed a decrease in maltose concentration over $72 \mathrm{~h}$ of incubation.

Furthermore, the detected range of fructan DP (up to 15) was similar to the results reported in other studies. An average DP of five makes up 50\% of the total fructan content in whole-wheat flour, with reported values up to DP 15 [14], whereas Haskå, Nyman, and Andersson [18] found the highest DP of 19. The fingerprint analysis of graminan-type fructans supported the results. Strains with a high degree of fructan hydrolysis showed intensively reduced peak heights in the HPAE chromatograms. Since high molecular weight fructans affect the enzymatic quantification stronger than smaller ones, only a moderate correlation can be expected.

\subsection{Fructan Degradation Potential of Different Yeast Strains}

For T. delbrueckii and Kl. marxianus MULC 30016, the highest potential to degrade fructans was observed in the model system and in the whole-wheat flour fermentation experiment. These findings are consistent with previous results [1] that have reported a $90 \%$ degradation of fructans by Kl. marxianus CBS6014 during bread making. In addition, Kl. marxianus MULC 30016 exhibits the potential to degrade fructose, which is released due to fructan depolymerisation to fructose and glucose. As described in other studies [31], S. cerevisiae showed a decreased potential to ferment fructans compared to the already mentioned yeast species. These findings concur with those of our study, as $S$. cerevisiae isolated from Austrian traditional sourdough had a degradation potential of $18 \%$ within $6 \mathrm{~h}$ of incubation, whereas after $72 \mathrm{~h}$, a decrease of $54 \%$ in the fructan content was achieved within the model system. However, within the whole-wheat flour experimental setup, a decrease in total fructans (until DP 15) of up to $83 \%$ was determined. As discussed previously, the marginal growth should be responsible for a low fructan conversion, whereas inoculation with similar yeasts counts inhibited this problematic circumstance.

In contrast, other sourdough-relevant yeasts proved to be less applicable in reducing FODMAPs in the model system and in the whole-wheat flour experiment. In particular, P. kudriavzevii MUCL 29043, C. glabrata MUCL 51245, W. anomalus DSM 6766, T. pretoriensis MUCL 27827, and the sourdough isolates H. uvarum and C. lambica showed poor fructan degradation.

\section{Conclusions}

This study evaluated the FODMAP degradation ability of different typical sourdough yeasts in a simplified model and when using wheat flour as a matrix. Both systems were able to identify differences between the examined strains and showed high consistency. Only the poor growth of S. cerevisiae inhibited a higher reliability of the model system. Furthermore, the results regarding the conversion of other FODMAPs such as raffinose were generated by the model system. The fingerprints of fructans measured by HPAEC before and after fermentation supported the fructan degradation results. A comparison with data from other studies confirmed the findings of this study. Wheat bakery products with low FODMAP content might be suitable for IBS patients, but probably only to a very limited degree for individuals suffering from NCWS. The role of ATIs (amylase/trypsin inhibitors) in wheat products must also be considered for people with NCWS [6,7]. In addition, further studies are needed to demonstrate the degradation of FODMAPs in the consumed product, in this case, sourdough fermented bread.

In summary, the results of this study clearly demonstrated the potential of several yeast strains, especially S. cerevisiae and T. delbrueckii, isolated from traditional sourdough, to strongly reduce 
FODMAPs, and particularly, the fructan content during fermentation. Furthermore, these yeasts exhibited superior $\mathrm{CO}_{2}$ production, which revealed their potential to produce wheat bread with improved leavening characteristics. Therefore, this study might aid to explore the potential of several yeasts to produce a low FODMAP diet with palatable food. However, typical sourdoughs contain a wide range of different lactic acid bacteria and yeasts. Thus, further research on their synergistic effects should be conducted. In particular, the ability of LAB to lower $\mathrm{pH}$, thus supporting yeast invertase activity, is of great interest [17]. Summing up, the purpose of this study was to generate basic data about the potential of single yeast strains to reduce FODMAPs. Based on our findings, more applied research studies should be performed to produce wheat breads suitable for patients with IBS or NCWS.

Supplementary Materials: Supplementary materials are available online at http:/ /www.mdpi.com/2072-6643/ 10/9/1247/s1. Table S1: Utilized yeast species, source of isolation, and determination of identification by MALDI-TOF MS and \% similarity with the accession number of the closest relative by blastn received by $16 S$ rDNA sequencing, Table S2: Determination of $\log$ CFU g ${ }^{-1}$ of 13 different yeast strains on YG-agar (Merck, Darmstadt, Germany), with incubation conditions of $25^{\circ} \mathrm{C}$ for $48 \mathrm{~h}$, Figure S1: Determination of the gas building capacity of (A) Saccharomyces cerevisiae, (B) Torulaspora delbrueckii MUCL 51211 and (C) Kluyveromyces marxianus MUCL 30016 by fermentation locks according to Einhorn, Figure S2: HPAEC-PAD analysis with CarboPac PAD $210250 \times 2 \mathrm{~mm}$ and eluent $\mathrm{A}(100 \mathrm{mM} \mathrm{NaOH})$ and B $(100 \mathrm{mM} \mathrm{NaOH} / 500 \mathrm{mM}$ NaAcetate $)$ to determine glucose, sucrose, fructose, raffinose, maltose, and fructans (max. DP 8) metabolism by Kluyveromyces marxianus MUCL 30016 at point 0 , after $6 \mathrm{~h}, 24 \mathrm{~h}, 48 \mathrm{~h}$, and $72 \mathrm{~h}$ of fermentation, Figure S3: HPAEC-PAD analysis of (A) Candida humilis MUCL 30041 and (B) Candida glabrata MUCL 51245, and (C) Pichia kudriavzevii MUCL 29043 showing the initial fructan presence (black line) and after $495 \mathrm{~min}$ of fermentation at $25^{\circ} \mathrm{C}$ (blue line), with a low conversion rate of fructan, Figure S4: HPAEC-PAD fructan fingerprint analysis of (A) Torulaspora delbrueckii MUCL 51211, (B) Saccharomyces cerevisiae-baker's yeast, (C) Kluyveromyces marxianus MUCL 30016, (D) Wickerhamomyces anomalus DSM 6766, (E) Torulaspora pretoriensis MUCL 27827, (F) Candida lambica-sourdough isolate, and (G) Hanseniaspora uvarum-sourdough isolate, showing the initial fructan presence (blue line) and after $8 \mathrm{~h} 15 \mathrm{~min}$ of fermentation (black line) at $25^{\circ} \mathrm{C}$, with moderate degradation of fructans, Figure S5: HPAEC-PAD analysis of (A) Kazachstania exigua MUCL 52365, (B) Saccharomyces cerevisiae-sourdough isolate, and (C) Torulaspora delbrueckii-sourdough isolate, showing the initial fructan presence (blue line) and after $8 \mathrm{~h} 15 \mathrm{~min}$ of fermentation (black line) at $25^{\circ} \mathrm{C}$, with the highest degradation of fructans.

Author Contributions: The authors V.F., K.J.D. and S.D. conceived and designed the methodology and conceptualization. V.F. performed the microbiological analysis, L.-M.C. and S.D. performed the HPAE-PAD analysis, and the method according to the AOAC 999.3 standard. V.F. and S.D. conducted data analysis and wrote the initial draft of the manuscript. K.J.D. and S.D. reviewed the final paper. V.F. wrote the final paper and S.D. submitted it. All authors approved the final manuscript.

Funding: This work is part of the ID-wheat project, which is supported by the Austrian Research Promotion Agency FFG (Project-Nr. 858540).

Acknowledgments: This study was supported by the project funded by FFG (Austrian Research Promotion Agency) “ID_WHEAT" (FFG project No: 858540). The Arnold wheat samples were kindly provided by E. Reiter from the Institute for Animal Nutrition and Feed of AGES (Austrian Agency for Health and Food Safety). The authors thank the EQ BOKU VIBT GmbH-Center for Preservation and Aseptic Processing for providing the MALDI-TOF mass spectrometer for yeast identification.

Conflicts of Interest: The authors declare no conflict of interest.

\section{References}

1. Struyf, N.; Vandewiele, H.; Herrera-Malaver, B.; Verspreet, J.; Verstrepen, K.J.; Courtin, C.M. Kluyveromyces marxianus yeast enables the production of low fodmap whole wheat breads. Food Microbiol. 2018, 76, $135-145$. [CrossRef] [PubMed]

2. Hungin, A.P.S.; Whorwell, P.J.; Tack, J.; Mearin, F. The prevalence, patterns and impact of irritable bowel syndrome: An international survey of 40,000 subjects. Aliment. Pharmacol. Therapeut. 2003, 17, 643-650. [CrossRef]

3. Lacy, B.E.; Patel, N.K. Rome criteria and a diagnostic approach to irritable bowel syndrome. J. Clin. Med. 2017, 6, 99. [CrossRef] [PubMed]

4. Canavan, C.; West, J.; Card, T. The epidemiology of irritable bowel syndrome. Clin. Epidemiol. 2014, 6, 71-80. [PubMed] 
5. Laatikainen, R.; Koskenpato, J.; Hongisto, S.M.; Loponen, J.; Poussa, T.; Huang, X.; Sontag-Strohm, T.; Salmenkari, H.; Korpela, R. Pilot study: Comparison of sourdough wheat bread and yeast-fermented wheat bread in individuals with wheat sensitivity and irritable bowel syndrome. Nutrients 2017, 9, 1215. [CrossRef] [PubMed]

6. D'Amico, S.; Call, L.; Edlmayr, M.; Marzak, S.; Jäger, H.; Domig, K.; Reiter, E.; Grausgruber, H. Characterization of non-gluten proteins in Austrian wheat varieties from 19th century until today. Cereal Technol. 2018, 2, 4-12.

7. Catassi, C.; Alaedini, A.; Bojarski, C.; Bonaz, B.; Bouma, G.; Carroccio, A.; Castillejo, G.; De Magistris, L.; Dieterich, W.; Di Liberto, D.; et al. The overlapping area of non-celiac gluten sensitivity (NCGS) and wheat-sensitive irritable bowel syndrome (IBS): An update. Nutrients 2017, 9, 1268. [CrossRef] [PubMed]

8. Catassi, G.; Lionetti, E.; Gatti, S.; Catassi, C. The low fodmap diet: Many question marks for a catchy acronym. Nutrients 2017, 9, 292. [CrossRef] [PubMed]

9. Gibson, P.R.; Shepherd, S.J. Evidence-based dietary management of functional gastrointestinal symptoms: The fodmap approach. J. Gastroenterol. Hepatol. 2010, 25, 252-258. [CrossRef] [PubMed]

10. Eswaran, S.; Farida, J.P.; Green, J.; Miller, J.D.; Chey, W.D. Nutrition in the management of gastrointestinal diseases and disorders: The evidence for the low fodmap diet. Curr. Opin. Pharmacol. 2017, 37, 151-157. [CrossRef] [PubMed]

11. Altobelli, E.; Del Negro, V.; Angeletti, P.M.; Latella, G. Low-fodmap diet improves irritable bowel syndrome symptoms: A meta-analysis. Nutrients 2017, 9, 940. [CrossRef] [PubMed]

12. De Roest, R.H.; Dobbs, B.R.; Chapman, B.A.; Batman, B.; O’Brien, L.A.; Leeper, J.A.; Hebblethwaite, C.R.; Gearry, R.B. The low fodmap diet improves gastrointestinal symptoms in patients with irritable bowel syndrome: A prospective study. Int. J. Clin. Pract. 2013, 67, 895-903. [CrossRef] [PubMed]

13. Verspreet, J.; Cimini, S.; Vergauwen, R.; Dornez, E.; Locato, V.; Le Roy, K.; De Gara, L.; Van den Ende, W.; Delcour, J.A.; Courtin, C.M. Fructan metabolism in developing wheat (Triticum aestivum L.) kernels. Plant Cell Physiol. 2013, 54, 2047-2057. [CrossRef] [PubMed]

14. Verspreet, J.; Dornez, E.; Van den Ende, W.; Delcour, J.A.; Courtin, C.M. Cereal grain fructans: Structure, variability and potential health effects. Trends Food Sci. Technol. 2015, 43, 32-42. [CrossRef]

15. Skodje, G.I.; Sarna, V.K.; Minelle, I.H.; Rolfsen, K.L.; Muir, J.G.; Gibson, P.R.; Veierod, M.B.; Henriksen, C.; Lundin, K.E.A. Fructan, rather than gluten, induces symptoms in patients with self-reported non-celiac gluten sensitivity. Gastroenterology 2018, 154, 529-539. [CrossRef] [PubMed]

16. Laatikainen, R.; Koskenpato, J.; Hongisto, S.M.; Loponen, J.; Poussa, T.; Hillilä, M.; Korpela, R. Randomised clinical trial: Low-fodmap rye bread vs. Regular rye bread to relieve the symptoms of irritable bowel syndrome. Aliment. Pharmacol. Therapeut. 2016, 44, 460-470. [CrossRef] [PubMed]

17. Struyf, N.; Laurent, J.; Lefevere, B.; Verspreet, J.; Verstrepen, K.J.; Courtin, C.M. Establishing the relative importance of damaged starch and fructan as sources of fermentable sugars in wheat flour and whole meal bread dough fermentations. Food Chem. 2017, 218, 89-98. [CrossRef] [PubMed]

18. Haskå, L.; Nyman, M.; Andersson, R. Distribution and characterisation of fructan in wheat milling fractions. J. Cereal Sci. 2008, 48, 768-774. [CrossRef]

19. Lewis, D.H. Nomenclature and diagrammatic representation of oligomeric fructans-A paper for discussion. New Phytol. 1993, 124, 583-594. [CrossRef]

20. Oku, T.; Nakamura, S. Digestion, absorption, fermentation, and metabolism of functional sugar substitutes and their available energy. Pure Appl. Chem. 2002, 74, 1253. [CrossRef]

21. Ziegler, J.U.; Steiner, D.; Longin, C.F.H.; Würschum, T.; Schweiggert, R.M.; Carle, R. Wheat and the irritable bowel syndrome-Fodmap levels of modern and ancient species and their retention during bread making. J. Funct. Foods 2016, 25, 257-266. [CrossRef]

22. Knez, M.; Abbott, C.; Stangoulis, J.C.R. Changes in the content of fructans and arabinoxylans during baking processes of leavened and unleavened breads. Eur. Food Res. Technol. 2014, 239, 803-811. [CrossRef]

23. Andersson, R.; Fransson, G.; Tietjen, M.; Åman, P. Content and molecular-weight distribution of dietary fiber components in whole-grain rye flour and bread. J. Agric. Food Chem. 2009, 57, 2004-2008. [CrossRef] [PubMed]

24. Loponen, J.; Ganzle, M.G. Use of sourdough in low fodmap baking. Foods 2018, 7, 96. [CrossRef] [PubMed] 
25. Lopez, H.W.; Duclos, V.; Coudray, C.; Krespine, V.; Feillet-Coudray, C.; Messager, A.; Demigné, C.; Rémésy, C. Making bread with sourdough improves mineral bioavailability from reconstituted whole wheat flour in rats. Nutrition 2003, 19, 524-530. [CrossRef]

26. Gobbetti, M.; Rizzello, C.G.; Di Cagno, R.; De Angelis, M. How the sourdough may affect the functional features of leavened baked goods. Food Microbiol. 2014, 37, 30-40. [CrossRef] [PubMed]

27. Brandt, M.J.; Gänzle, P.D.M. Handbuch Sauerteig; Behr: Santa Ana, CA, USA, 2006.

28. De Vuyst, L.; Harth, H.; Van Kerrebroeck, S.; Leroy, F. Yeast diversity of sourdoughs and associated metabolic properties and functionalities. Int. J. Food Microbiol. 2016, 239, 26-34. [CrossRef] [PubMed]

29. Lhomme, E.; Lattanzi, A.; Dousset, X.; Minervini, F.; De Angelis, M.; Lacaze, G.; Onno, B.; Gobbetti, M. Lactic acid bacterium and yeast microbiotas of sixteen French traditional sourdoughs. Int. J. Food Microbiol. 2015, 215, 161-170. [CrossRef] [PubMed]

30. Huys, G.; Daniel, H.-M.; De Vuyst, L. Taxonomy and biodiversity of sourdough yeasts and lactic acid bacteria. In Handbook on Sourdough Biotechnology; Gobbetti, M., Gänzle, M., Eds.; Springer: Boston, MA, USA, 2013; pp. 105-154.

31. Struyf, N.; Laurent, J.; Verspreet, J.; Verstrepen, K.J.; Courtin, C.M. Saccharomyces cerevisiae and kluyveromyces marxianus cocultures allow reduction of fermentable oligo-, di-, and monosaccharides and polyols levels in whole wheat bread. J. Agric. Food Chem. 2017, 65, 8704-8713. [CrossRef] [PubMed]

32. Waite, J.G.; Jones, J.M.; Yousef, A.E. Isolation and identification of spoilage microorganisms using food-based media combined with rDNA sequencing: Ranch dressing as a model food. Food Microbiol. 2009, 26, 235-239. [CrossRef] [PubMed]

33. Call, L.-M.; D’Amico, S.; Grausgruber, H.; Schönlechner, R. Fruktane in alten und neuen österreichischen Weizensorten. Getreide Mehl und Brot 2018, 1, 2-6.

34. Verspreet, J.; Dornez, E.; Delcour, J.A.; Harrison, S.J.; Courtin, C.M. Purification of wheat grain fructans from wheat bran. J. Cereal Sci. 2015, 65, 57-59. [CrossRef]

35. van de Wiele, T.; Boon, N.; Possemiers, S.; Jacobs, H.; Verstraete, W. Inulin-type fructans of longer degree of polymerization exert more pronounced in vitro prebiotic effects. J. Appl. Microbiol. 2007, 102, 452-460. [CrossRef] [PubMed]

36. Kurtzman, C.P.; Fell, J.W.; Boekhout, T.; Robert, V. Chapter 7-Methods for isolation, phenotypic characterization and maintenance of yeasts. In The Yeasts, 5th ed.; Kurtzman, C.P., Fell, J.W., Boekhout, T., Eds.; Elsevier: London, UK, 2011; pp. 87-110.

37. Tester, R.F.; Karkalas, J. Carbohydrates I classification and properties. In Encyclopedia of Food Sciences and Nutrition, 2nd ed.; Caballero, B., Ed.; Academic Press: Oxford, UK, 2003; pp. 862-875.

38. Nonklang, S.; Abdel-Banat, B.M.; Cha-aim, K.; Moonjai, N.; Hoshida, H.; Limtong, S.; Yamada, M.; Akada, R. High-temperature ethanol fermentation and transformation with linear DNA in the thermotolerant yeast kluyveromyces marxianus dmku3-1042. Appl. Environ. Microbiol. 2008, 74, 7514-7521. [CrossRef] [PubMed]

39. Michel, M.; Kopecka, J.; Meier-Dornberg, T.; Zarnkow, M.; Jacob, F.; Hutzler, M. Screening for new brewing yeasts in the non-saccharomyces sector with Torulaspora delbrueckii as model. Yeast 2016, 33, 129-144. [CrossRef] [PubMed]

40. Lertwattanasakul, N.; Rodrussamee, N.; Suprayogi; Limtong, S.; Thanonkeo, P.; Kosaka, T.; Yamada, M. Utilization capability of sucrose, raffinose and inulin and its less-sensitiveness to glucose repression in thermotolerant yeast Kluyveromyces marxianus DMKU 3-1042. AMB Express 2011, 1, 2-11. [CrossRef] [PubMed]

(C) 2018 by the authors. Licensee MDPI, Basel, Switzerland. This article is an open access article distributed under the terms and conditions of the Creative Commons Attribution (CC BY) license (http:/ / creativecommons.org/licenses/by/4.0/). 